\title{
Regular Polytopes of Nearly Full Rank: Addendum
}

\author{
Peter McMullen
}

Received: 5 November 2012 / Accepted: 1 February 2013 / Published online: 23 February 2013

(C) Springer Science+Business Media New York 2013

\begin{abstract}
A small family of regular polytopes of nearly full rank was omitted from the earlier paper with this title. This omission is rectified here.
\end{abstract}

Keywords Abstract regular polytope, Realization, Nearly full rank

Mathematics Subject Classification (2010) Primary, 51M20

\section{Introduction}

At the end of [2], we expressed the hope that our enumeration of the regular polytopes of nearly full rank was then complete. In retrospect, it is fortunate that we did not make an absolute claim for completeness; it turns out that we overlooked a family which is closely related to others that we did describe. In this note, we shall repair the omission.

Perhaps a brief word is in order about how we found the new family. In writing [5], it has seemed useful to present a wider range of illustrations of realization theory than we felt appropriate to put in papers. In particular, the new techniques of $[3,4]$ have enabled us to describe realization domains of polytopes which were out of reach of the theory in [6, Chap. 5]. One example (which actually has a rather complicated realization domain) is the dual $J_{5}^{\delta}$ of the polytope that in [2, Sect. 13] we called $J_{5}$; this six-dimensional regular polytope of rank 5 has 270 vertices, which it shares with the difference body $D\left(2_{21}\right)$ of the Gosset polytope $2_{21}$. At an early stage of looking at this polytope, we discovered that the corresponding abstract regular polytope had

\footnotetext{
P. McMullen ( $ه)$

University College London, Gower Street, London, WC1E 6BT, UK

e-mail: p.mcmullen@ucl.ac.uk
} 
another six-dimensional realization $K_{5}$ (in the notation of Sect. 2), with the 27 vertices of $2_{21}$ itself. This realization is our starting point.

It is worth pointing out as well that a further opportunity to come across this family was missed. In [1, Sect. 10], we eliminated two possible candidates as regular polytopes of full rank. What we failed to appreciate subsequently is that the facet of the six-dimensional case, namely $K_{5}$, was actually a polytope.

\section{The New Family}

The new family of regular polytopes (or apeirotopes) is derived by twisting the diagram below by an improper inner automorphism. If we omit the rightmost node (and corresponding branch), then we obtain the diagram for the polytope called $G_{s+1,3}^{\pi}$ in [2, Sect. 11]; it is the Petrial of the polytope $G_{s+1,3}$ of the first Gosset class, whose details are given in [2, Proposition 11.1]. (We have changed the index from the original for future convenience.)

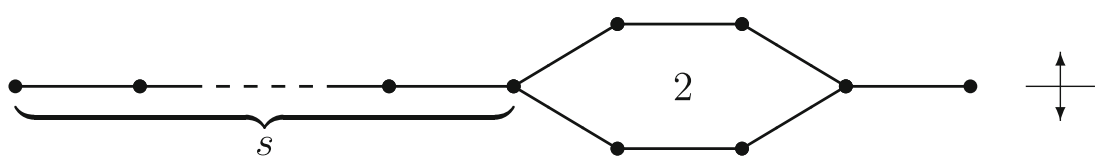

It is clear that we cannot extend the diagram by any more nodes to the right, because we would then obtain the diagram of the infinite group $\left[3^{2,2,2}\right]$ as a subdiagram. However, as we shall shortly see, we can allow any $s \leq 2$, in spite of the fact that there is again an 'infinite' subdiagram; we encountered such a situation several times in [2, Sect. 12].

So, let us draw the diagram in the case $s=2$, now with labels attached which indicate the corresponding generators of the symmetry group $\mathbf{G}$.

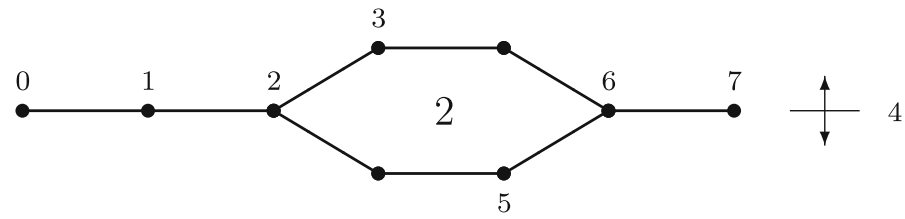

For the present purposes, the best way to list the generators $R_{j}$ of $\mathbf{G}$ is by giving the equations of their mirrors, in terms of coordinate vectors $\left(\xi_{1}, \ldots, \xi_{8}\right) \in \mathbb{E}^{8}$. They are

$$
R_{j}: \begin{cases}\xi_{1}+\xi_{8}=2, & \text { if } j=0, \\ \xi_{1}+\cdots+\xi_{8}=0, & \text { if } j=1, \\ \xi_{2}+\xi_{7}=0, & \text { if } j=2, \\ \xi_{2}=\xi_{3}, & \text { if } j=3, \\ \xi_{1}=\xi_{8}, \xi_{2}=\xi_{7}, \xi_{3}=\xi_{6}, \xi_{4}=\xi_{5}, & \text { if } j=4, \\ \xi_{5}=\xi_{6}, & \text { if } j=5, \\ \xi_{4}=\xi_{5}, & \text { if } j=6, \\ \xi_{1}+\cdots+\xi_{4}=\xi_{5}+\cdots+\xi_{8}, & \text { if } j=7 .\end{cases}
$$


Thus $R_{4}$ is the diagram twist, which just reverses the order of the coordinates $\xi_{1}, \ldots, \xi_{8}$.

Of course, as we know from [6, Chap. 2], to show that we do obtain polytopes (rather than pre-polytopes), we must verify the intersection property

$$
\left\langle R_{i} \mid i \in \mathrm{J}\right\rangle \cap\left\langle R_{i} \mid i \in \mathrm{K}\right\rangle=\left\langle R_{i} \mid i \in \mathrm{J} \cap \mathrm{K}\right\rangle
$$

for all subsets $\mathrm{J}, \mathrm{K} \subseteq\{0, \ldots, 7\}$. However, the geometric picture given by the generators makes this straightforward, if a little tedious.

For $r=5, \ldots, 8$, the symmetry group of the general member $K_{r}$ of the family is

$$
\mathbf{K}_{r}:=\left\langle R_{8-r}, \ldots, R_{7}\right\rangle .
$$

For $r=5,6,7, K_{r}$ is a (finite) $(r+1)$-dimensional regular polytope of rank $r$, and thus of nearly full rank; similarly, $K_{8}$ is an eight-dimensional apeirotope of nearly full rank.

The initial vertex of $K_{8}$ is the origin $o$; which $R_{0}$ takes into the initial vertex $\left(2,0^{6}, 2\right)$ of the vertex-figure $K_{7}$; as usual in this context, $\alpha^{k}$ denotes a string $\alpha, \ldots, \alpha$ of length $k$. Under the group $\mathbf{G}_{0}=\mathbf{K}_{7}$ of the vertex-figure, we obtain all permutations with an even number of changes of sign of $\left(2,2,0^{6}\right)$ and $\left(1^{8}\right)$, namely, the vertex-set of the Gosset polytope $4_{21}$. Thus $K_{8}$ has the vertices of the semi-regular tiling $5_{21}$ of $\mathbb{E}^{8}$.

More generally, the $r$-coface (that is, coface of rank $r$ ) $K_{r}$ has the same vertices as $(r-3)_{21}$. Moreover, as we said above, the facet of $K_{r}$ is the Petrial $G_{r-5,3}^{\pi}$ of the regular polytope $G_{r-5,3}$ of the first Gosset class. We pointed out in [2, Sect. 11] that $G_{33}^{\pi}$ is an apeirotope, whose facets are themselves apeirotopes; these are actually of type $J_{6}$ of Sect. 13 , rather than of type $A_{6}$ of Sect. 12 as mistakenly asserted. Hence $K_{8}$ even has ridges which are apeirotopes.

So far as $K_{5}$ is concerned, its group is obtained from that of $J_{5}^{\delta}$ by changing the sign of the diagram twist $T$ (that is, replace the mirror $T$ by its orthogonal complement $T^{\perp}$ ); this changes a proper outer automorphism to an improper inner one. Since such replacements were used quite often in [2, Sect. 12], this makes the fact that $K_{5}$ was overlooked even less excusable.

Let us add one comment about $K_{7}$. In spite of the apparent symmetry of the diagram, $K_{7}$ is not self-dual; indeed, like each of the polytopes $K_{r}$, it has no geometric dual. However, just as with other cases, if we reverse the order of the generators $R_{1}, \ldots, R_{7}$ and change the sign of the twist $R_{4}$, then we obtain the symmetry group of another copy of $K_{7}$ (or, rather, the same copy, but with different initial vertex and so on).

\section{References}

1. McMullen, P.: Regular polytopes of full rank. Discrete Comput. Geom. 32, 1-35 (2004)

2. McMullen, P.: Regular polytopes of nearly full rank. Discrete Comput. Geom. 46, 660-703 (2011)

3. McMullen, P.: Realizations of regular polytopes, III. Aequationes Math. 82, 35-63 (2011)

4. McMullen, P.: Realizations of regular polytopes, IV. Aequationes Math. (in press)

5. McMullen, P.: Geometric Regular Polytopes (in preparation)

6. McMullen P., Schulte, E.: Abstract Regular Polytopes. Encyclopedia of Mathematics and Its Applications, No. 92. Cambridge University Press, Cambridge (2002) 